\title{
"iMollis o fortis femina?": la ambigüedad del personaje de Briseida en la Historia Troyana Polimétrica
}

\author{
"¿Mollis or fortis femina?": the ambiguity of Briseida's character in the Historia \\ Troyana Polimétrica
}

\author{
Agustina Miguens \\ agustinamm@hotmail.com \\ Universidad de Buenos Aires, Argentina
}

Recepción: 30 Octubre 2020

Aprobación: 24 Abril 2021

Publicación: 03 Mayo 2021

Cita sugerida: Miguens, A. (2021). “ $¿$ Mollis o fortis femina?": la ambigüedad del personaje de Briseida en la Historia Troyana Polimétrica. Olivar, 21(33), e096. https://doi.org/10.24215/18524478e 096
Resumen: El presente trabajo examinará la representación del personaje de Briseida en la Historia troyana polimétrica (HTP), tomando en consideración las diferencias con su fuente directa, el Roman de Troie, y las interpretaciones que podrían haber suscitado en sus contextos de circulación, la corte angevina del siglo XII y la castellana del XIV, respectivamente. Los temas principales de las obras son las armas y el amor, los cuales se ven sintetizados en el personaje de Briseida, la cautiva enamorada de Troylo, pero que luego lo olvida en favor del griego Diomedes. Aunque la trama amorosa tiene un importante desarrollo, en consonancia con los códigos del amor cortés, la volubilidad de Briseida es severamente reprochada por el narrador en un pasaje con una clara influencia del discurso misógino medieval. Allí se retoma el tópico de la mollis femina, la mujer de voluntad débil y cambiante, en contraste con la fortis femina, fuerte y constante. Esta última aparece identificada con la referencia a "una rica dama de rico rey”, que, según el texto y el contexto de recepción, puede interpretarse tanto como una dedicatoria a una dama de la corte, como a la Virgen María, modelo de virtud femenina.

Palabras clave: Historia Troyana Polimétrica, Roman de Troie, Mujeres, Virtud femenina.

Abstract: This work examines the representation of Briseida in the Historia troyana polimétrica (HTP), considering the differences with its source, the Roman de Troie, and the interpretations that they could have aroused in their circulation contexts, the XII ${ }^{\text {th }}$ century Angevin court in one case, and the $\mathrm{XIV}^{\text {th }}$ century Castillian court in the other. The main topics of these texts are Militia and Amor, synthesized in Briseida's character, the war prisoner who falls in love with Troylus, but then forgets him for the Greek Diomedes. Even though the relationships are developed according to the rules of courtly love, Briseida's change of heart is severely rejected by the narrator in a passage with a notable influence from the medieval misogynous speech. There, the voluble and frail woman (mollis femina) is contrasted with the stable and strong-willed fortis femina, identified with a "riche dame de riche rei". This reference can be interpreted, according to the different texts and contexts of reception, as a dedicatory either to a woman of the court, or to the Virgin Mary, the most perfect model of female virtue.

Keywords: Historia Troyana Polimétrica, Roman de Troie, Women, Female virtue. 


\section{INTRODUCCIÓN}

La Historia Troyana Polimétrica $(H T P)^{1}$ es una de las traducciones castellanas ${ }^{2}$ del Roman de Troie. Se transmite a través de dos manuscritos facticios: el M (10.146 de la Biblioteca Nacional de Madrid), datado a fines del siglo XIV, y el E (L.jj.16 de la Biblioteca de El Escorial), del siglo XV. De autor anónimo, la fecha de composición del texto se estima entre fines del siglo XIII y la mitad del siglo XIV. El Roman de Troie (RT) de Benoît de Sainte-Maure, escrito en francés antiguo a hacia 1165, es el texto más relevante e influyente sobre la guerra de Troya del período medieval, ya que circuló ampliamente en forma de copias o traducciones, como la Historia destructionis Troiae del italiano Guido delle Colonne, versión latina y abreviada que circuló aún más que su hipotexto. El relato de Benoît hunde sus raíces en la tradición anti homérica de la materia troyana, en especial en el De excidio Troiae Historia (del siglo V o VI) del falso mitógrafo Dares frigio, que relata la caída de Troya desde el punto de vista de un supuesto cronista del bando troyano. Benoît amplifica el escueto raconto de su fuente hasta alcanzar una extensión de más de 30.000 versos y adapta las fuentes tardoantiguas actualizándolas al modo de representación del naciente roman francés. Por esta razón, se lo considera dentro del género de los romans antiques, junto con el Roman de Thèbes, el Roman d'Énéas y el Roman d'Alexandre.

Tanto en el $R T$ como en la HTP los tópicos de las armas y el amor resultan centrales y se ven sintetizados en la trama de Briseida a partir de sus amores sucesivos por dos caballeros, Troylo y Diomedes, y los vínculos que se establecen entre ellos y los avatares de la guerra. Podemos trazar un paralelismo y una inversión entre su historia y la de Elena ya que, mientras el rapto de esta última constituye el casus belli, la devolución Briseida de los troyanos a los griegos preanuncia el desenlace de la guerra. Los amores de Briseida, invención de Benoît, son puestos en primer plano en la $H T P$ en seis de los once poemas entrelazados en la prosa y tuvieron una enorme influencia posterior, tal como lo atestiguan Il Filostrato de Boccaccio, Troylus and Criseyde de Chaucer y la obra de teatro homónima de Shakespeare. A pesar del importante desarrollo narrativo del enamoramiento, en sintonía con los códigos del amor cortés, y del retrato positivo de Briseida, a quien se describe como una mujer bella y una amante sincera, el cambio en sus afectos es severamente reprochado por el narrador en un pasaje con una clara influencia del discurso misógino medieval. Allí se retoma el tópico de la mollisfemina, la mujer de voluntad débil y cambiante, en contraste con la fortisfemina, fuerte y constante. Esta última aparece identificada con la referencia a "una rica dama de rico rey", que, según el texto y el contexto de recepción, puede interpretarse tanto como una dedicatoria a una dama de la corte, Leonor de Aquitania probablemente en el caso del $R T$, como a la Virgen María, modelo de virtud femenina.

Para los propósitos de este trabajo, nos centraremos en el personaje de Briseida, sus dos amores, Troylo y Diomedes, y el paralelismo entre esta tríada y la de Elena, Menelao y Paris. Se examinará especialmente la representación del personaje de Briseida en el Roman de Troie y en la HTP, sus similitudes y diferencias, y las interpretaciones que podría haber suscitado en distintos contextos de circulación, la corte angevina, por un lado, y la castellana, por el otro.

\section{LAS ARMAS Y EL AMOR}

Para comenzar, es importante señalar que la combinación entre los tópicos del amor y de la guerra es una de las características fundantes del roman y que estos se manifiestan de manera conjunta e inseparable:

Lo novelesco es esta fusión entre el amor y la guerra, fusión de naturaleza arquetípica, es decir, que forma una pareja de opuestos donde uno nunca se separa de su contrario, y que se enraíza en el suelo de la psicología suprapersonal, transhistórica, 
incluso abismal. Pero, como expresión literaria, es el resultado de una elaboración histórica: está vinculada con el personaje del caballero medieval, tal como se lo concibió a partir del siglo XII hasta el XVI, lo novelesco solo puede aparecer con la revolución afectiva que el hombre conoció con la cortesía, del mismo modo que con la consideración de la guerra como una proeza individual y estética. (Stanesco-Zink, 1992, p. 21)

En particular, Lumiansky (1958) y Adler (1960) han señalado la imbricación entre los tópicos de Militia y Amor como el pilar sobre el que se edifica la estructura del Roman de Troie. Lumiansky (1958) destaca cuatro parejas, -Jasón y Medea, Elena y Paris, Troilo y Briseida, y Aquiles y Polixena-, cuyas tramas además están perfectamente entrelazadas con el desarrollo de la historia de la guerra. De estas parejas, todas excepto la de Troilo y Briseida, están presentes en el texto de Dares, aunque apenas mencionadas. La amplificación del tópico amoroso corresponde al autor francés, a quien también se atribuye la invención del nudo amoroso entre Troilo y Briseida (Kelly, 1995). ${ }^{3}$

En efecto, la cuestión del intercambio de mujeres ${ }^{4}$ funciona como un hilo conductor que vincula los relatos de las cuatro parejas con el desarrollo de la Guerra de Troya y con el traslado simbólico del poder (traslatio imperii). En cuanto a lo primero, en el $R T$ se describen una serie de afrentas que enemistan a griegos y troyanos desde la expedición de Jasón y los Argonautas en busca del vellocino de oro. Cuando estos pasan por la costa troyana, el rey Laomedón los ofende profundamente al negarles el hospedaje. Como respuesta, Hércules junta un ejército de caudillos griegos para efectuar la primera destrucción de Troya, en la que mata al rey y a sus hijos y rapta a su hija, quien es tomada como barragana por Telamón. Príamo jura venganza por la destrucción de la ciudad, la matanza de sus familiares y sobre todo por el rapto y la deshonra de su hermana. El motivo de la dama raptada actualiza la afrenta contra los troyanos y, asimismo, establece un paralelismo con el rapto de Elena a manos de Paris, señalado como el desencadenante directo de la guerra y la segunda destrucción de la ciudad. En el mito, suele ser Casandra quien denuncia que Elena causará la caída de Troya, mientras que el resto de la familia real la recibe como una hija más. El buen recibimiento que le da Príamo puede indicar, a la luz del rapto de su hermana, que constituye para él una venganza simbólica. Del mismo modo, los amores de la cautiva Briseida por Troylo y Diomedes y luego el amor arrebatador que siente Aquiles por Polixena, una de las hijas de Príamo, también forman parte de la cadena de intercambio de mujeres.

No obstante, entre las cuatro parejas se destacan especialmente los paralelismos entre Elena y Briseida, ambas con dos amantes. Estas, además, son las dos parejas presentes en la $H T P$, que corresponde a la traducción de los versos 5703 a 15567 del $R T$ en el caso del manuscrito M, y de los versos 13822 a 15567 en el ms. E, con numerosas lagunas internas. El rapto de Elena, que luego deviene ejemplo de amor conyugal con Paris, marca en primer lugar la supremacía troyana. Croizy-Naquet, siguiendo a E. Baumgartner, señala que Elena es la pieza que le faltaba a Troya para alcanzar su apogeo, la cumbre de la perfección como ciudad y civilización, al conjugar riquezas, la grandeza de sus muros, sus palacios y templos y la destreza de su caballería con la conquista del amor y la belleza (1990, p. 90). Sin embargo, esta unión conduce, paradójicamente, a la ruina de la ciudad.

En el plano bélico, mientras Héctor resulta un personaje central del $R T$, en consonancia con la perspectiva troyana que impone el influjo de Dares, su rivalidad con Aquiles como máximo caudillo griego se encuentra atenuada en comparación con el relato homérico. En su lugar, cobran relevancia otros líderes griegos y, entre ellos, Diomedes y sus enfrentamientos con Troylo. El destino de este último presenta correlatos importantes con su patria de origen, no solo por su condición de príncipe troyano, sino también por las resonancias semánticas de su nombre, que significa "pequeña Troya”. En este sentido, su desengaño amoroso con Briseida y la rivalidad personal con Diomedes adquieren una interpretación a nivel político-militar.

En cuanto a Briseida, su abandono de Troylo por Diomedes, -junto con otros eventos como las profecías y la traición de Calcas-, anticipa el desenlace de la guerra a favor de los griegos y la restitución de Elena a su primer esposo Menelao. Por lo tanto, a nivel simbólico, los movimientos tanto de Elena como Briseida marcan el derrotero de la supremacía militar entre los bandos, y ambas parejas reproducen los eventos de la guerra como un microcosmos. 


\section{BRISEIDA: ¿MOLLIS O FORTIS FEMINA?}

¿El cambio en los afectos de Briseida debe ser interpretado como una falta en su carácter? ¿Se trata del pecado de lujuria, que sobrevuela el relato encarnado en las figuras de Briseida y Elena y que causa la caída de Troya, según la interpretación cristiana que prevaleció durante el período medieval? ¿ O bien, en este horizonte también puede haber lugar para una perspectiva compasiva con estas mujeres, víctimas de un destino y unas condiciones militares adversas en un mundo dominado por hombres? En la literatura medieval la cuestión de la virtud femenina se abordaba predominantemente desde una óptica masculina y una postura misógina. ${ }^{5}$ Kelly (1995) retoma la oposición entre la mollis y la fortis femina, la mujer débil y cambiante en contraste con la estable y fuerte, basada en la moral tradicional judeo cristiana, que promueve a la mujer como virgen o como esposa fiel. Señala que funciona como un tópico o lugar común en la producción textual en torno a la corte angevina y que Benoît, como voz narrativa, interviene para juzgar a Briseida en un pasaje que retoma la tradición misógina y que puede servir como modelo de la actitud esperable por parte de la audiencia:

Therese Latzke (1989) has shown that the fortis femina or fortis mulier -the woman with fortitude- is a commonplace analogous to the puer senex, especially in the Anglo-Angevin courts of pre- and early vernacular literature. The fortis femina designates the woman who rises above the place assigned her in medieval misogyny, a place Benoit as narrator rehearses for his audience in evaluating Briseida's conduct. (Kelly, 1995, p. 228)

No obstante, la censura a la actitud de Briseida no es una conclusión que se desprenda necesariamente de la narración de los hechos, y quizás esto motivó la intervención del narrador. Por lo tanto, para facilitar el análisis de esta cuestión proponemos distinguir tres niveles de sentido que se despliegan en tensión bajo la cuidadosa estructura textual de Benoît. Primero, un nivel narrativo, en el que tomamos en consideración los retratos de los personajes y el relato de los hechos. En segundo lugar, un nivel evaluativo, en el que se introduce el discurso misógino que reprueba la volubilidad de Briseida. Por último, un nivel que se proyecta hacia lo contextual y que se vincula con la inserción de la dedicatoria.

\section{1- BRISEIDA EN EL RELATO DE LOS HECHOS}

Comenzaremos por el nivel narrativo, para lo cual seguiremos el análisis que realiza Lumiansky (1954) sobre cómo la historia amorosa de Troylo y Briseida se entreteje en el $R T$ y luego examinaremos las similitudes y diferencias con la HTP.

Primeramente, Benoît presenta los infortunios amorosos de Troylo en el resumen ${ }^{6}$ de los hechos que le sigue al prólogo, lo cual da tempranas muestras de la importancia de esta línea narrativa. Luego, incluye un retrato suyo en el que se destacan su belleza física y su cortesía. Por su parte, se describe a Briseida en el catálogo de personajes griegos como una joven hermosa con un rasgo particular: las cejas un poco juntas. ${ }^{7}$ Más adelante, el padre de Briseida, el adivino Calcas, consulta el oráculo de Apolo y se entera de la futura victoria griega, por lo que decide desertar del bando troyano. El siguiente punto relevante de la trama es cuando se produce el primer duelo singular entre Troylo y Diomedes, en el que este último pierde su caballo, pero luego le arrebata el suyo a Troylo logrando, de este modo, obtener la ventaja. En un segundo duelo, Troylo demuestra su cortesía al desmontar del caballo cuando su oponente queda a pie, en contraste con la actitud que había tenido Diomedes (Lumiansky, 1954, p. 729). Como veremos, el leitmotiv del caballo reaparecerá conectado con la historia amorosa cuando Diomedes le envía como regalo a Briseida el caballo ganado a Troylo. Podemos deducir que funciona como símbolo de dominio, tanto sobre el contrincante como sobre la dama.

Luego de todas estas menciones que lo preludian, el lector se entera de la relación entre Troylo y Briseida cuando están a punto de ser separados. ${ }^{8}$ Ella es una prisionera de guerra entre los troyanos, aunque de alta alcurnia. Cuando surge la oportunidad de un intercambio de rehenes, Calcas solicita que devuelvan a su hija al bando griego. En ese momento se desatan los lamentos de los amantes, quienes hacen uso del discurso 
directo para expresar sus penas ante la perspectiva de la separación. La postura del narrador en este punto también es compasiva con ellos.

Apenas después de la separación comienza el cortejo de Diomedes, quien conduce a Briseida al campamento griego. Mientras Diomedes se enamora a primera vista (vv. 13542-44 del $R T$ ), Briseida opone resistencia y se enamora gradualmente, lo cual contribuye a crear la imagen de una elección consciente y práctica, tal como afirma Kelly: "Her gradus amoris leads to a conscious, even practical, choice made in conformity with her and her family's destiny as revealed by the gods" (Kelly, 1995, p. 236-237). La historia de Briseida es la de una amante sincera con el corazón dividido, pero que finalmente cederá ante lo inevitable.

En este contexto, se produce el tercer duelo 9 entre Troylo y Diomedes, esta vez mediado por el conflicto amoroso. El griego se cobra el caballo de Troylo y se lo envía a Briseida como trofeo, pero ella lo rechaza y le reprocha que sea capaz de lastimar a un ser aún querido por ella. Además, le dice que no puede odiarlo, puesto que no sería adecuado odiar a alguien que la ama, pero que no le devolverá sus afectos. Como respuesta, se produce la enfermedad de amor de Diomedes. ${ }^{10}$ Briseida se rinde únicamente cuando el caballero se humilla ante ella y se somete por completo a su voluntad.

En el siguiente duelo, Diomedes ostenta una manga de su dama en el hasta de la lanza. Los caballeros se baten con ímpetu. Troylo le reprocha el haberle arrebatado el amor de Briseida y afirma públicamente que le será infiel también a él. A esto le sigue un monólogo en el que Briseida lamenta la volubilidad de su propio corazón y manifiesta conciencia de su mala reputación, pero promete ser fiel a Diomedes. En un pasaje a modo de conclusión (vv. 20666-20682), Benoît expone la total desilusión de Troylo con el conjunto de las mujeres y explica que el buen nombre de Briseida está arruinado (Lumiansky, 1954, p. 732).

En todo este desarrollo es visible la influencia del discurso cortés sobre el amor en pleno desarrollo a mediados del siglo XII. En particular, la reticencia inicial de la dama, el largo servicio de amor por parte del caballero y su humillación frente a la domina, la obligatoriedad en la retribución de ese servicio en términos de atenciones, en paralelo a la reciprocidad del vínculo vasallático feudal, la utilización de ciertos símbolos, como la manga en el pendón del caballero (Schnell, 1989). Lo transgresor es que la dama cambie de afectos, pero el código de la cortesía se aplica a las dos relaciones, que no son simultáneas sino sucesivas.

En la HTP no conservamos los pasajes correspondientes al prólogo ni los retratos, pero el núcleo del triángulo amoroso es objeto de una importante amplificación y destacado a través de la elección de la lírica. Los lamentos de Troylo y de Briseida por su separación se suceden en las poesías V y VI, culminando en la despedida de los amantes a dos voces en la poesía VII. Es notable la influencia sobre estas composiciones de la lírica provenzal y gallego portuguesa, tanto en cuanto a estructura formal, como a recursos y tópicos. Deyermond (1975) destaca la relación entre los poemas V a X con la lírica del amor cortés, la influencia del alba de origen provenzal sobre la poesía VII y las reminiscencias a las cantigas de amigo de origen gallego portugués, en consonancia con el desarrollo del fin'amor o amor cortés. Sirva como ilustración la sumisión del amante a la dama en la poesía V: "El mi bien, el mi seso / la mi vida viçiosa, / todo lo tiene preso / la mi señor fermosa" (Menéndez Pidal ed., 1934, p. 128)11 y el deseo de mejorar como caballero por amor: "sy yo soy esforçado / o ardit o apuesto / por ella lo soy todo" (p. 129). En esta instancia no se pone en duda que el amor de la pareja es absolutamente genuino: se aman el uno al otro "mas que a ssy" (p. 128 y p. 132).

Si bien los poemas suscitaron tempranamente la atención de la crítica, aún no se ha prestado suficiente atención a los versos que evidencian que, cada uno de los amantes por su lado (poemas V y VI) y luego juntos (poema VII), analizan su situación y sopesan todas sus opciones racionalmente. De hecho, es significativo a nivel estructural cómo el poema VII recoge los sentimientos y los argumentos que Troylo y Briseida habían expresado antes por separado y los pone en diálogo a través de un contrapunto a dos voces en el que se podría identificar la influencia de la dialéctica, la cual habría formado parte de los saberes clericales del autor.

Por más que Troylo resiente la directiva de su padre, quien ha ordenado la devolución de Briseida, en su monólogo rechaza la posibilidad de traicionarlo: "mas en esto so loco / sy por una donzella que echan de la tierra, / maguer que la cobrase, / feziese tan gran yerra / que traydor me tornase” (p. 131). En soledad, Briseida 
también reconoce que poco se puede hacer contra la orden del rey: "Mas ya quequier que nos cueste / convien nos de yr ayna, / pues lo el rrey por bien tiene, / non ay hi al de fazer" (p. 133). No obstante, cuando ambos se reúnen para un último encuentro antes de la despedida, el problema vuelve a surgir. Briseida acusa al mismo Troylo de no defenderla frente al soberano troyano: "En vos yo rrebtar derecho / fago e muy gran rrazon, / ca bien se que, sy pesase / mucho a vos de la mi yda, / non cuydo que me echase / Priamo atan escarnida" (p. 136). A través de estas palabras, Briseida le plantea un dilema entre su lealtad como amante cortés hacia ella y la que le debe a su rey y padre. Pero Troylo se rehúsa a traicionar a su rey: "Mas señor ¿commo creredes / que yo quisiese la muerte / del rrey Priamo?” (p. 137). Es decir, que entre el amor y el deber, Troylo escoge este último. Entre lágrimas y besos, los dos examinan racionalmente las posibilidades que les quedan, si Briseida podría ocultarse o si Troylo podría ir con ella, pero ambas conducen a la traición:

Aquella noche a su grado / por siempre les durarie; / mill rraazones han fablado / por veer commo podrien / por qual guisa o por qual arte / por ellos seer desfecho / por engenio o por arte / aquel tan esquivo fecho. / Pensavan de la donzella / esconderse sy podrie, / o yrse Troylo con ella; / traicion dezie que serie: / sy el a griegos fuyese, / dar lo yan por alevoso; / e sy ella se escondiese, / saldria el rrey por mintroso (p. 139).

La conclusión que se desprende es que ambos ponen el deber y la lealtad por encima de su amor y aceptan así su destino trágico. Cierra la poesía de despedida un pasaje donde aparece la voz del poeta quien, siguiendo a Benoît, se muestra conmovido por la pareja: “iDios, que fuerte que pecaron / dios, que grande mal fezieron / quantos les esto guisaron / e los en esto metieron!” (p. 139). Esta postura del narrador, que se compadece de los amantes después de tres largos y conmovedores pasajes líricos en los que se expresan su amor, contrasta fuertemente con el pasaje misógino que aparece más adelante.

Haywood señala que las repetidas alusiones al pecado, sobre todo en la muletilla "mal pecado", apuntan, en última instancia, al "amor adúltero y concupiscente" (1998, p. 219) que causa la caída de Troya, y lo analiza en relación tanto con Briseida como con el pecado de lujuria en el que habría incurrido Paris al secuestrar a Elena (1998, p. 217). No obstante, en este caso la referencia al pecado se refiere no a los amantes, sino a los responsables de su separación. Si bien no resulta fácil identificar la referencia, unos versos antes Troylo señala a Priamo y a Calcas como los culpables, pero también establece una concatenación de acciones que apuntan a la guerra misma como causa última de sus desgracias (p. 137). ${ }^{12}$

Como cierre de la poesía VII, se incluye una anticipación de que la separación provocará en Troylo una ira vengativa contra los griegos que causará gran mortandad: "por esta coyta tan maña / fue Troylo el infante / cogiendo tan brava saña / contra griegos e tan fuerte, / que el mesmo, por sus manos / vengandose, dio la muerte / mas d'a mill griegos loçanos" (p. 139-140). Podemos vincular esta reacción violenta con la interpretación de Adler de que, en el Roman de Troie, los tópicos de la Militia et Amor adquieren un matiz trágico ya que no se subliman, sino que entrañan fuerzas destructivas: "Rather than to generate Amor, the political and military situation (Militia) threatens to destroy Amor. On the other hand, Amor, even though it may stir Militia, tends to undermine the very foundation of Militia" (Adler, 1960, p. 15). Adler comprende el interjuego de Militia et Amor como dinámicas de raigambre platónica, esto es, que el amor hace mejores caballeros y que un buen caballero será un buen amante. No obstante, en el caso de los amores de Troylo y Briseida, el lector es testigo de la destrucción de un amor que era inicialmente genuino y conmovedor, a causa de las exigencias de la Militia, es decir, el deber de lealtad de Troylo a su rey y a su pueblo (Adler, 1960, p. 20). ${ }^{13}$

En el momento en el que los amantes se separan, se inserta el pasaje misógino, el cual será objeto de análisis en el próximo apartado. Luego continúa el relato de los hechos: cómo Diomedes conduce a Briseida al campamento griego, cómo se enamora de ella a primera vista y cómo comienza a cortejarla (p. 149-152). En este punto, ella rechaza sus atenciones, en consonancia con el código cortés, pero se aclara que lo hace no solo para resguardar su buen nombre, -al respecto afirma que: "non es derecho nin rrazon, nin serie tenida a buen prez, se yo fablar agora convusco de amor, ca vos mesmo o qualquiera otro me ternia ende por muy vil e por muy ligera" (p. 152)-, sino también porque aún transita el duelo por Troylo: "so ora en tan grand duelo, non 
ha ningund sabor de amor nin de alegria aver nin plazer, ca yo dexe a muy buen amigo e muy leal, e bien se que nunca lo cobrare" (p. 153). En este punto se empieza a percibir una discrepancia entre el discurso de Briseida y sus acciones, ya que aunque no acepta públicamente el homenaje de Diomedes, tampoco lo rechaza cuando este la toma de la mano sin que nadie los vea (p. 154). Briseida sigue manifestando públicamente su amor a Troylo cuando amonesta a su padre por traidor (p. 155), y cuando rechaza el caballo de Troylo que Diomedes le regala como trofeo de guerra, pero le permite entrar a su tienda. Únicamente cuando Diomedes muestra todos los síntomas de la enfermedad de amor (amor hereos), por no ser correspondido, es que Briseida se rinde y le obsequia una manga para que lleve como pendón y seña pública de su amor (p. 196). En esto, la $H T P$ sigue fielmente el desarrollo en el $R T$.

Lumiansky compara el delicado desarrollo de los amores de Briseida en la obra de Benoît, el cual incluye diálogos y monólogos que reflejan una progresión psicológica en los personajes, con la adaptación de Guido Colonne al latín, con abreviaciones y supresiones que modifican el significado que se desprende de la narración. Mientras la perspectiva de Benoit es mayormente compasiva con los amantes, la de Guido presenta la relación amorosa en términos reprobables (Lumiansky, 1954, p. 730). ${ }^{14}$ Tanto en el RT como en la HTP se despliegan las sucesivas emociones y razonamientos de Briseida. La imagen que se desprende es la de una mujer cautiva que se amolda a las circunstancias en un mundo determinado por las decisiones masculinas, los sucesos de la guerra y el destino dictado por los dioses. Siguiendo a Lumiansky, se trata de un personaje con un carácter complejo y a la vez práctico: "the choice topic for psychological speculation in Benoît's version of this love story is of course Briseida, who in her long soliloquy and elsewhere exhibits her complex mixture of downto-earth realism and evasive rationalization" (Lumiansky, 1954, p. 733). En cambio, en el relato de Guido su accionar carece del adecuado concatenamiento. Allí, Briseida es un ejemplo de la natural inconstancia femenina y por lo tanto, la principal responsable de las miserias de Troylo. En síntesis, la visión es más intensamente misógina en Guido y esta será la que influya especialmente en las adaptaciones subsiguientes del relato, de Boccaccio a Shakespeare, mientras que tanto en el $R T$ como en la HTP la visión que se desprende del relato de los hechos es principalmente conmovedora y compasiva con los amantes.

\section{2- BriseidA COMO MOLLIS FEMINA}

Mientras a partir del desarrollo de los hechos podemos observar un desarrollo psicológico en el personaje de Briseida y una actitud pragmática y realista, aunque en línea con ciertas formas osificadas del amor cortés, justo en el momento de la separación con Troylo se inserta, tanto en el $R T$ como en la HTP, un pasaje en el que se rechaza explícitamente su actitud y se la califica como una mujer débil e inconstante (mollis femina). En el mismo, se detiene la acción narrativa y se desarrolla un largo excursus didáctico, que debe mucho a la larga tradición misógina medieval, en el que la voz narrativa adoctrina sobre la volubilidad femenina apelando directamente a los receptores. De este modo, el narrador se postula a sí mismo como un receptor modelo, capaz de interpretar el relato de manera moral. Abrevando en referencias del Antiguo Testamento, de la Biblia y los Padres de la Iglesia, pero también en auctoritates de origen pagano como Ovidio, de gran influencia en el discurso erótico medieval, se rechaza el natural supuestamente pecaminoso, concupiscente, engañoso y cambiante de las mujeres, personificado en la figura de Briseida y cuyo modelo bíblico es Eva. En contraste, se promueve un modelo de virtud femenina basado en la castidad y la fidelidad conyugal, cuyo mayor epígono es la Virgen María. El modelo de mujer de corazón “débil”, inconstante en sus sentimientos, desleal e indigna de confianza corresponde a la mollis femina, en tanto que el ejemplo de constancia, virtud y fortaleza de espíritu se identifica con la fortis femina.

El pasaje comienza con una prolepsis que anuncia que Briseida olvidará a Troylo tan rápido como se enamoró de él y que sus sentimientos experimentarán un vuelco rotundo, lo que se expresa a través del conector adversativo y frases paralelas que marcan una antítesis: "Mas commo quier que Breyseda oviese tan grand amor e tan gran coyta commo esta que veedes aquí, de aquí adelante este amor tan grande ayna 
se partira; (...) E quando cresçio en gran sazon, descreçera en poco tiempo” (p. 146). ${ }^{15}$ Tanto cuanto antes conmovía el amor sincero entre ella y Troylo, ahora indigna su rápida traición. A continuación, alecciona sobre el carácter inconstante y engañoso de Briseida y lo hace extensivo a todo el género femenino: "sabed que toda mogier..." (p. 147) ${ }^{16}$ No obstante, reconoce que esto sucede cuando la dama tiene otro pretendiente masculino que la corteja, idea que sintetiza mediante una cita de autoridad ovidiana: "creo que es verdat lo que escrivio Ovidio, que fue muy sabidor en estas cosas, quando dixo: "subcesore novo vincitur omnis amor, que quiere dezir: "todo amor es vençido por el nuevo entendedor"” (p. 147). Luego, insiste en varios lugares comunes del discurso misógino, ${ }^{17}$ utilizando recursos como el refranero popular: "sy ela mogier con el un ojo lora, con el otro rrie" (p. 147), la hipérbole: "mas quanto aman en seys años o siete, todo lo dexan olvidar en tres dias" (p. 147), "çierto soy e bien creo que sy yo oviese çient lenguas e con todas podiese fablar, non podria dezir la quarta parte de las maldades que an las que son malas dellas” (p. 147) y la enumeración de defectos. Estos funcionan como argumentos misóginos, entre los que se inserta la conclusión de que no es aconsejable fiarse de las mujeres: "e por ende faz muy grand locura quien las crey, ca mudan mucho ayna su coraçon, e en poca de ora es la mays sesuda tornada loca e sandia” (p. 147).

A continuación y en contraste con esta acumulación de vituperios que caracterizarían al género femenino, se introduce una alabanza a una "rica dama de rico rey" (148), ${ }^{18}$ la excepción a la regla. Mientras en el $R T$ este sintagma hace referencia con bastante probabilidad a Leonor de Aquitania y constituiría una dedicatoria, en la HTP se reinterpreta como una alusión a la Virgen María. ${ }^{19}$ A esta se adjudican un cúmulo de virtudes vinculadas a la santidad y al borramiento del pecado: "una tan solamente en que ha tanta de apostura e en quien ha tanta de bondat e de santidat e tanta nobleza, que por los sus bienes descreçe el mal que començava a cresçer en los coraçones de los onbres" (p. 148). El amor terrenal pasional es contrapuesto al amor divino, el caballero amigo es reemplazado por Jesucristo, el cual garantiza alegría eterna en lugar de una pasajera: "sienpre aya alegria esta, que mantiene todo tienpo a su amigo e su señor Jehsu Cristo leal amor, e todas las otras fagan lo que ovieren e an sabor de la semejar" (p. 148). La Virgen es presentada, entonces, como fortis mulier y modelo de virtud femenina.

Para cerrar la digresión moralizante, ${ }^{20}$ retoma algunos argumentos de la primera parte, como que las mujeres son en su mayoría cambiantes y ligeras: "Salamon, que fue sabidor e muy entendido en todas cosas, dixo dellas: "quien fallar mogier fuerte, de loor a dios por ende"; esto dixo el, por que las mogieres non son fuertes, ante son muy ligeras" (p. 148), señala que los varones que cortejan insistentemente también son igual o más culpables que las damas seducidas: "non son tanto de rrebtar commo los onbres cuydan, ca a las mas dellas faze gelo fazer el quexo de los rrogadores que porfian tanto e duran tanto en su porfia, que los han por fuerça de otorgar" (p. 148) y concluye, a modo de síntesis y recurriendo nuevamente al discurso paremiológico, que hallar una mujer virtuosa es algo extraordinario: "E por ende, mogier casta e fermosa, sy puede ser fallada en ninguna guisa, mas debe ser preçiada que piedra preçiosa” (p. 148). La estructura del pasaje resulta ser concéntrica, ya que el contenido de mayor relevancia, el elogio a la Virgen, se incluye en el medio, precedida y sucedida por la censura a la supuesta inconstancia femenina.

\section{3- LA DEDICATORIA Y LOS CONTEXTOS DE RECEPCióN}

Hemos mencionado la referencia a una "rica dama de rico rey", identificada en la $H T P$ con la Virgen. No obstante, en el $R T$ de Benoît de Sainte-Maure, la crítica está de acuerdo en relacionarla con una dama de la corte a quien pueda haber sido dedicado el poema. Aunque no se mencionen nombres, la candidata más probable es Leonor de Aquitania, reina consorte de Inglaterra en segundas nupcias con Enrique II Plantagenet, como sostienen Constans (1912, p. 189-190) y Cowper (1930, p. 380). Este último señala como fundamentos: el dialecto del poema, escrito en francés antiguo, la fama de Leonor como mecenas y que el epíteto "rico rey" era atribuido especialmente a Enrique, en contraste con Luis VII, rey de Francia y primer 
marido de Leonor, quien era notoriamente más pobre (Cowper, 1930, p. 380). ${ }^{21}$ Otro factor es la hipótesis de que el autor proviene efectivamente de Sainte-Maure, cerca de Poitiers, sede de la corte de los duques de Aquitania. Esta era afamada por sus costumbres más alegres y menos solemnes que las de sus señores, los reyes de Francia, y por el desarrollo de la lírica provenzal, cultivada por los mismos nobles, como Guillermo IX de Poitiers, abuelo de Leonor. Ella también fue una importante patrona de trovadores y artistas, al igual Enrique II. ${ }^{22}$

En caso de tratarse efectivamente de una dedicatoria dirigida a Leonor de Aquitania, esta no deja de ser problemática. Se inserta en un pasaje que marca un supuesto contraste entre la "rica dama de rico rey" y la actitud voluble de Briseida y la mayoría de las mujeres. Los atributos que se le atribuyen son la bondad, el valor, la mesura, la santidad, la nobleza, la generosidad, la belleza y la alegría (vv. 13457-70). ${ }^{23}$ Ahora bien, la comparación con el personaje de Briseida no arroja un contraste tan claro, debido a que Leonor misma tuvo dos maridos. Su primer matrimonio con Luis, que dura quince años y produce dos hijas, es finalmente anulado después de muchas desavenencias entre los cónyuges y rumores de infidelidad promovidos por los detractores de Leonor. Si bien la unión con Enrique parece haber sido menos conflictiva, por lo menos hasta la década de 1170, estos detalles sobre la reina eran de público conocimiento y no habrían podido ser pasados por alto por una audiencia cortesana. Del mismo modo que Briseida y a Elena resultan piezas importantes en relación a la traslatio imperii, el "traslado" de Leonor como esposa representa la transferencia del poder que le brindan las extensas y ricas tierras del Ducado de Aquitania, del reino de Francia al de Inglaterra. ${ }^{24}$ En este sentido, ¿puede identificarse una fina ironía por detrás de la dedicatoria y alabanza de Benoît?

En cuanto a la HTP, si bien la dedicatoria se reinterpreta como dirigida a la Virgen, el énfasis en los triángulos amorosos no deja de traer reminiscencias problemáticas con respecto al momento de circulación del texto. Se han propuesto dos posibles fechas de composición para esta obra. Menéndez Pidal (1934) propuso datarla hacia el año 1270, cuando se estaban redactando la General Estoria y la Estoria de España en el taller alfonsí, pero la mayor parte de la crítica actual ${ }^{25}$ la ubica a mediados del siglo XIV, coetánea a la Crónica Troyana (CT) de Alfonso XI (1350). En todo caso, la HTP se conserva copiada en dos manuscritos facticios de entre mediados del siglo XIV y fines del XV, el M. (10.146 de la BNM) ${ }^{26}$ y el E. (esc. L.jj.16), ${ }^{27}$ lo cual prueba la circulación y el interés que suscitaba la materia troyana en este período turbulento para la historia de Castilla.

Nuria Larrea Velasco, quien realizó la edición más reciente del texto (2012), conjetura que pueda ser obra del entorno cortesano del rey Alfonso XI y que incluso pueda tener la misma autoría que el Poema de Alfonso Onceno, por una serie de similitudes temáticas, estilísticas y estructurales. Observa que la historia de Elena y sus "dos maridos", y podemos agregar a Briseida y sus amantes, podría identificarse con Alfonso XI, quien abandona a su legítima esposa María de Portugal en favor de su amante Leonor de Guzmán, con quien tiene no menos de diez hijos (Larrea Velasco, 2012, p. 24). El adulterio fue escandaloso, ya que mientras María se recluye lejos de la corte, doña Leonor ocupa su lugar públicamente. La venganza emprendida por María y su hijo Pedro I contra la amante y sus hijos luego de la muerte del monarca provocó una guerra civil que culminó con el ascenso al trono de Enrique II de Trastámara.

Por último, no debemos olvidar que la materia troyana tenía una importancia en términos genealógicos y simbólicos para las monarquías europeas en cuyas cortes se compusieron y adaptaron estos relatos. El vínculo entre personajes de la leyenda troyana y personajes aristocráticos y regios ha estado presente desde las primeras referencias en ámbito ibérico. Helena de Carlos Villamarín señala los versos del Carmen Campidoctoris (siglo XI) donde se comparan las hazañas del Cid con las de Paris y Eneas como ejemplo de esta temprana voluntad de filiación (1998, p. 90). El interés de las dinastías reinantes, algunas recientemente establecidas y con la presión constante de una nobleza potencialmente rebelde, por adquirir legitimidad a través del establecimiento de genealogías semi legendarias que se remontaban a los relatos de la antigüedad, está atestiguado en Francia por la proliferación de crónicas familiares, de literatura épica y de literatura 
genealógica, como la Historia regum Britanniae de Godofredo de Monmouth, Le Roman de Rou y el Roman de Brut de Wace o las Chroniques des ducs de Normandie (Bloch, 2019, p. 94) ${ }^{28}$, obras del siglo XII y aproximadamente coetáneas al Roman de Troie. Del mismo modo, en la convulsionada Castilla del siglo XIV puede establecerse un correlato entre la eclosión de relatos de tema troyano con una voluntad de afianzamiento de la autoridad regia, especialmente en el caso del reinado de Alfonso XI. Este da un impulso renovador a la caballería, con el objetivo de mantenerla bajo su mando, al reanudar la Reconquista, lo que permite el reparto de tierras y el ascenso de nuevos hidalgos, y al crear la Orden de la Banda, que se encontraba bajo control directo de la persona del rey.

La paradoja y la tensión se producen porque al mismo tiempo que estos relatos contribuyen a cimentar un modelo de caballería y a construir la idea de traslatio imperii de la Antigüedad hacia los reinos medievales de occidente, introducen la idea subversiva del amor pasional como fuerza potencialmente desestabilizadora y destructiva, sintetizada en las figuras femeninas de Elena y de Briseida.

\section{ConCLUSIÓN}

En este trabajo hemos buscado desplegar una serie de capas de sentido en tensión, producto de las transformaciones que implica la adaptación medieval de un relato que tiene su origen en la Antigedad Clásica y que se reelabora en el período Tardoantiguo. En cuanto al $R T$, la composición se realiza en el contexto de la corte angevina en el siglo XII, donde la moral cristiana coexiste con la producción de trovadores y poetas que celebran el amor cortés; en el caso de la HTP, probablemente en la corte de Alfonso XI, heredera de la producción textual alfonsí, en la que se yuxtaponen el discurso misógino y la literatura caballeresca, la cual conjuga las armas y el amor.

En los dos textos, el $R T$ y su traducción en la $H T P$, la conexión entre las armas y el amor muestran su cara más trágica y devastadora. En ellos todavía es visible la concepción clásica del destino designado por los dioses, en el que los personajes juegan un rol que está establecido de antemano. Asimismo, se perciben huellas del amor cortés, ya que Benoît probablemente haya sido un poeta con saberes clericales vinculado a la corte de Leonor de Aquitania, mecenas de la poesía trovadoresca. Esta forma de discurso redescubre un amor pasional, íntimo y subjetivo, que muchas veces transgrede desde lo ficcional las normas de fidelidad conyugal. Georges Duby señala que el fin' amor tiene un desarrollo ficcional y por ende autónomo, como literatura de evasión (2012, p. 16-17). Sin embargo, aunque el amor cortés en ocasiones cantó al adulterio, también se proponía regular la sexualidad de las personas de la corte, tanto de las mujeres casadas, que debían mantenerse fieles por la importancia de la herencia para el linaje, como de los caballeros, que solo podían solazarse con sirvientas, no con alguien de su misma condición: "Su función fue promulgar un código de comportamiento cuyas prescripciones apuntaban a limitar en la aristocracia militar los estragos de un desenfreno sexual irreprimible" (Duby, 2012, p. 24). Por ello, el mismo código cortesano sobre el amor dictaba, paradójicamente, la celebración y la contención de los impulsos eróticos de los miembros de la corte en un nudo contradictorio irreductible.

Por otro lado, la materia antigua de estos relatos se vincula con la historia, cuya finalidad es edificante. En consonancia con la interpretación predominante de la leyenda troyana desde un punto de vista religioso cristiano, como alegoría del pecado de lujuria y de soberbia que lleva a la caída de la ciudad, el autor incluye un pasaje moralizante que adoctrina en contra de la fragilidad y volubilidad femenina, asimilando a Briseida a la pecadora Eva y haciendo uso de los argumentos del discurso misógino medieval. Por sobre el común de las mujeres, se eleva la "rica dama de rico rey", Leonor de Aquitania o, ya en un plano supraterrenal, la Virgen María, en uno y otro texto.

El resultado de estas capas de sentido en tensión es un relato complejo en el que hasta el amor más perfecto, como el de Elena y Paris, lleva al sufrimiento y la destrucción. En relación a Briseida, cuanto más conmovedor es su idilio con Troylo, más indignante resulta su traición. Su volubilidad es censurada especialmente desde 
el punto de vista moral cristiano. No obstante, esta reprobación no se desprende del relato mismo de los hechos, en el que se desarrolla la psicología del personaje, sus emociones y razones, así como su impotencia ante decisiones tomadas por líderes varones en el contexto de la guerra. Allí se muestra a un personaje realista, práctico y que respeta el código cortés del amor en sus dos relaciones.

A su vez, la reprobación de su conducta también entra en tensión con el complejo contexto de recepción y circulación de las obras, compuesto por un entorno cortesano y los posibles comitentes. Tanto Leonor de Aquitania como Alfonso XI tuvieron uniones político-matrimoniales conflictivas y cuestionadas. Mientras la condición femenina de Leonor la hacía más susceptible a las críticas por sus dos matrimonios y los rumores de infidelidad, el adulterio masculino en sí mismo no era repudiado, sino que en el caso de Alfonso XI el inconveniente radicaba en que despreció públicamente a su esposa legítima y elevó casi al rango de segunda esposa a su amante, quien además le dio muchos más hijos. Esto generó un conflicto sucesorio, porque las facciones nobiliarias, siempre dispuestas a la conjura, tomaron partido a la muerte del rey por los herederos de una u otra mujer, Pedro I o por Enrique de Trastámara, lo que desembocó en una guerra civil.

Por lo tanto, la alabanza al comitente resulta un tópico y no deja de parecer una solución de compromiso que busca armonizar las contradicciones entre el relato de un triángulo amoroso trágico y conmovedor, la censura de las mujeres, de raigambre cristiana y misógina, y los ecos de sentido que podían producir los textos en sus contextos de circulación: ámbitos cortesanos en los que las uniones matrimoniales y las aventuras amorosas de los reyes y reinas resultaban una cuestión de Estado.

\section{ReFERENCiAS}

Adler, A. (1960). Militia et amor in the Roman de Troie. Romanische Forschungen, 72 (1/2), 14-29.

Benoît de Sainte-Maure. (1904-1912). Le Roman de Troie, (6 vols. ed. Léopold Constans). Paris: Librairie de FirminDidot et Cie.

Brownlee, M. S. (1979). Undetected Verses. Historia troyana en prosa y verso Romania, 100(398), 270-272.

Bloch, H. (2019). Etimologías y genealogías: teorías de la lengua, lazos de parentesco y género literario en el siglo XIII. En Basarte, A. y Cordo Russo, L. (comps.), Géneros literarios medievales (pp. 87-114). Buenos Aires: Eudeba.

Croizy-Naquet, C. (1990). La complainte d'Hélène dans le Roman de Troie (v. 22920-23011). Romania, tome 111 (441-442), 75-91.

De Carlos Villamarín, H. (1998). Algunas huellas de materia troyana en el Medievo hispano. En C. Leonardi (ed.), Gli Umanesimi Medievali. Atti del II Congreso dell" "Internationales Mittellateinerkomitee" (Firenza, 11-15 settembre 1993) (pp. 85-95). Firenze: SISMEL - Edizioni del Galuzzo.

Deyermond, A. (1975). Lyric traditions in non-lyrical genres. En Nitti, J. (ed.), Studies in Honor of Lloyd A. Kasten (39-52). Madison: Hispanic Seminary of Medieval Studies.

Dodds, E. R. (1997). Los griegos y lo irracional. Madrid: Alianza Editorial.

Duby, G. (2012). El modelo cortés. En Basarte (comp.) y Dumas (ed.), Nueve ensayos sobre el amor y la cortesía en la Edad Media (pp. 11-34). Buenos Aires: Editorial de la Facultad de Filosofía y Letras de la Universidad de Buenos Aires.

Faral, E. (1913). Chronologie des romans d'Énéas et de Troie. Revue des langues romanes 56, 129-147.

García Solalinde, A. (1916). Las versiones españolas del Roman de Troie. Revista de Filología Española, 3, 121-165.

Haywood, L. M. (1998). Al "mal pecado" de los troyanos: lírica y modos narrativos en la Historia troyana polimétrica. En Ward, A. (ed.), Actas del xii Congreso de la Asociación Internacional de Hispanistas (21-26 de agosto de 1995, Birmingham) (pp. 216-221, Vol. I). Birmingham: University of Birmingham \& Department of Hispanic Studies.

Kelly, D. (1995). The Invention of Briseida's Story in Benoît de Sainte-Maure's "Troie". Romance Philology, 48(3), 221-241. 
Larrea Velasco, N. (2012). Historia Troyana Polimétrica. Edición crítica (Tesis doctoral). Universidad Nacional de Educación a Distancia, Buenos Aires. Recuperado de: http://e-spacio.uned.es/fez/eserv/tesisuned:Filologia-Nl arrea/Documento.pdf

Lumiansky R. M. (1954). The Story of Troilus and Briseida according to Benoît and Guido. Speculum, 29(4), 727-733.

Lumiansky R. M. (1958). Structural Unity in Benoit's Roman de Troie. Romania, 79(315), 410-424.

Menéndez Pidal, R. y Varón Vallejo, E. (Eds.). (1934). Historia troyana en prosa y verso. Revista de Filología Española, Anejo XVIII. Madrid: Junta para la Ampliación de Estudios \& Centro de Estudios Históricos, VII-IL.

Peláez, L. (1996). La Historia troyana polimétrica: una tentativa de renovación de la épica culta. Olifant, 20(3-4), 235-248.

Schnell, R. (2012). El amor cortés como discurso cortés sobre el amor. En Basarte (comp.) y Dumas (ed.), Nueve ensayos sobre el amor y la cortesía en la Edad Media (pp. 321-424). Buenos Aires: Editorial de la Facultad de Filosofía y Letras de la Universidad de Buenos Aires.

Stanesco, M. y Zink, M. (1992). Histoire européenne du roman medieval: esquisses et perspectives. París: Presses Universitaires de France. Disponible en: https://gallica.bnf.fr/ark:/12148/bpt6k48142109/f17.image.texteIm age

\section{Notas}

1 Nombraremos estas obras a partir de sus siglas de aquí en más.

2 La otra es la Crónica Troyana o Versión de Alfonso XI, terminada de copiar en el ms. A (esc. H.j.6) en 1350 por pedido expreso del monarca. La crítica actual considera que ambas traducciones deben provenir de otra traducción anterior a una lengua romance ibérica occidental, sea el portugués, el gallego o el leonés.

3 Esta creación debe entenderse en los términos de las prácticas de composición medievales del siglo XII, tal como sostiene Douglas Kelly (1995): no es producto de la imaginación original de un individuo, sino que se basa en las técnicas de traducción (amplificación), composición tradicional (motivos) y la conjoncture o entrelazamiento de episodios en el roman. Sobre esta base, deben haber tenido importancia el motivo del intercambio de rehenes, la isotopía de las armas y el amor que estructura el poema y los otros modelos de parejas.

4 Mientras en el mito griego puede observarse el eco de un motivo folklórico (el rapto de la novia, de fuerte raigambre patriarcal), en el roman asistimos a la cristalización de dicho motivo como parte integral de la ficción caballeresca. De manera análoga se utilizan los motivos tradicionales celtas en el roman artúrico, separándolos de su trasfondo mítico original y dotándolos de un nuevo significado literario con un mayor grado de autonomía.

5 Hacia fines del siglo XIV se alzan otras voces en defensa de la virtud de las mujeres y en contienda con el discurso misógino. Estas se desarrollan en un plano mayormente retórico-discursivo, al que la crítica suele referirse como la "querelle desfemmes" o el debate sobre la virtud de las mujeres.

6 Versos 145-714 del $R T$. Todas las citas se realizarán a partir de la edición de Constans (1904-1912), por lo que a continuación se señalará, únicamente, número de verso.

7 Algunos críticos han señalado este rasgo como un defecto estético y lo han vinculado a la imperfección moral interna de Briseida (Kelly, 1995).

8 Versos 13261-13528 del $R T$.

9 Versos 14268-14385 del RT.

10 Versos 15001-15186 del $R T$.

11 Todas las citas de la $H T P$ se realizarán a partir de la edición de Menéndez Pidal (1934), por lo que a continuación solo se señalará el número de página o línea.

12 "Por mi mal, señor, fue dada / esta tregua en la batalla, / e por mal de mi pasaron / los griegos el mar aquende, / señor, pues que rrecabdaron / de pasar a vos allende, / e por mal el vuestro padre, / que vos dexo aca donzella / quando morio vuestra madre / non morio e fincase ella, / ca el a Troya non dexara / nin fuera por traydor dado / nin a mi non me matara / commo m'mato mal pecado" (Menéndez Pidal ed., 1934, p. 137).

13 "In the case of Briseida, Troilus and Diomedès, the political and military circumstances (Militia), like a consuming fire, badly scorch the very nature of Amor itself. Amor survives, but at the cost of heavy losses, the loss of Briseida's feelings for Troilus, the loss of Troilus' faith in women and in love (20664 f.), the loss of Briseida's self-respect” (Adler, 1960, p. 20).

14 "Where Benoit seems genuinely sympathetic with the lovers, and makes only brief mention of the universal falsity of women and of Briseida's future betrayal, Guido chooses to add a lengthy section reproaching Troilus for trusting Briseida 
and proclaiming that all women are fickle. Further, where Benoit gives a ring of sincerity to the grief of the lovers, Guido so exaggerates the situation that it becomes almost ludicrous" (Lumiansky, 1954, p. 730).

15 Líneas 1-10 de la146 de la HTP.

16 Líneas 1-11, p. 147 de la HTP.

17 Líneas 11-27, p. 147 de la HTP.

18 Líneas 28-33, p. 147 y 1-18, p. 148 de la HTP.

19 Esta confusión ya está presente en una familia de manuscritos tardíos del $R T$, unos eliminan la dedicatoria y otros la adjudican a la Virgen (Constans, 1912, p. 189-190). Alguno de ellos podría haber servido de base para el traductor ibérico.

20 Líneas 8-28, p. 148 de la HTP.

21 "Although no patron's name is mentioned, this dedication could be appropriate, as Constans said (VI, 189), only to the queen of France or the queen of England. His decision in favor of Eleanor rested on the dialect of the poem. But there are two other arguments in her favor: first, the allusions in the later lines of the dedication are rather obvious in view of Eleanor's reputation; and, second, the epithet of riche rei was generally applied to Henry and was at least implied by no less a person than Louis VII himself, according to Walter Map. On the other hand, Louis was notoriously por" (Cowper, 1930, p. 380).

22 Estas suposiciones contribuyen a fijar la fecha de composición del RT entre 1154, cuando Leonor anula su primer matrimonio con Luis VII y se desposa con Enrique II, y 1173, cuando es apresada en una torre por promover una rebelión de sus hijos contra su marido. En particular, la crítica considera más probable una fecha entre 1160 y 1170 , cuando finalizan una serie de campañas militares por la pacificación del reino, y cerca de 1165, porque estilísticamente parece posterior al Roman de Thèbes y al Énéas (Faral, 1913).

23 "De cest, veir, criem g'estre blasmez / De celé que tant a bontez / Que hautece a, pris e valor, / Honesté e sen e honor, / Bien e mesure e sainteé, / E noble largece e beauté; / En cui mesfait de dames maint / Sont par le bien de li esteint; / En cui tote science abonde, / A la cui n'est nule seconde / Que el mont seit de nule lei. / Riche dame de riche rei, / Senz mal, senz ire, senz tristece, / Poisseiz aveir toz jorz leece!" (Constans, 1912, p. 187).

24 A través de la unión con Enrique se conforma, en efecto, el Imperio Angevino, que abarcaba todo el occidente de la actual Francia, Inglaterra, Gales y parte de Irlanda, por lo que de hecho eran más poderosos que el rey de Francia, a pesar de rendirle homenaje por sus posesiones continentales.

25 Solalinde (1916), Haywood (1998), Peláez (1996) y Larrea Velasco (2012) se inclinan por una fecha más tardía, anterior o coetánea a la Crónica Troyana (1350).

$26 \mathrm{El} \mathrm{ms.} \mathrm{M} \mathrm{(10.146} \mathrm{de} \mathrm{la} \mathrm{BNM)} \mathrm{está} \mathrm{constituido} \mathrm{por} \mathrm{dos} \mathrm{núcleos} \mathrm{redacciones} \mathrm{distintos,} \mathrm{uno} \mathrm{de} \mathrm{mediados} \mathrm{del} \mathrm{siglo} \mathrm{XIV,}$ $\mathrm{Ma}$, que contiene la $C T$, interrumpida por el fragmento de la $H T P$, y uno de fines del siglo XV, $\mathrm{M} b$, que suple los folios perdidos de la parte antigua con una refundición de la $C T$ y capítulos tomados de la General Estoria alfonsí (García Solalinde, 1916, p.124).

27 El ms. E. (esc. L.jj.16), de fines del XIV, también presenta una redacción miscelánea que contiene la $C T$, la $H T P$ entre los folios 157b-180b y una traducción castellana de la Historia destructionis Troiae de Guido delle Colonne (García Solalinde, 1916, p. 125).

28 La crítica aún debate si la Chronique des ducs de Normandie es obra del mismo Benoît que escribió el Roman de Troie. 